\title{
Spectral and Morphological Studies of the Very High Energy Gamma-Ray Source 2HWC J1825-134
}

\author{
F. Salesa Greus ${ }^{* a}$, and S. Casanova ${ }^{a, b}$ for the HAWC Collaboration ${ }^{c \dagger}$ \\ ${ }^{a}$ Institute of Nuclear Physics, Polish Academy of Sciences \\ ul. Radzikowskiego 152, 31-342 Krakow, Poland \\ ${ }^{b}$ Max-Planck Institute for Nuclear Physics \\ Saupfercheckweg 1, Heidelberg, BW 69117, Germany \\ ${ }^{c}$ A complete list of authors and acknowledgments is available at \\ http://www.hawc-observatory.org/collaboration/icrc2019.php \\ E-mail: fsalesa@ifj.edu.pl, sabrina.casanova@ifj.edu.pl
}

\begin{abstract}
With an energy range from around $300 \mathrm{GeV}$ to more than $100 \mathrm{TeV}$, the High Altitude Water Cherenkov (HAWC) observatory is currently the most sensitive gamma-ray survey instrument in the TeV energy range. Recent HAWC data show that 2HWC J1825-134 is the brightest source above $56 \mathrm{TeV}$ in the HAWC field of view. The region of J1825 is complex and contains multiple astrophysical sources, e.g. pulsar wind nebulae, supernova remnants, and molecular clouds. The study of VHE galactic sources like 2HWC J1825-134 is crucial to understand the production and acceleration processes of cosmic rays in the Galaxy, and can clarify whether these sources can provide the power needed to sustain the galactic cosmic ray population up to the knee. In this contribution we will present the morphological analysis of 2HWC J1825-134, discussing the possible astrophysical counterparts of this source. We will also present the spectral analysis, comparing different scenarios of particle acceleration (hadronic/leptonic), and will provide an estimate of the associated cosmic-ray flux produced and the maximum acceleration energy.
\end{abstract}

36th International Cosmic Ray Conference -ICRC2019-

July 24th - August 1st, 2019

Madison, WI, U.S.A.

\footnotetext{
* Speaker.

${ }^{\dagger}$ for collaboration list see PoS(ICRC2019)1177
} 


\section{Introduction}

Cosmic rays (CRs) of energies around $\mathrm{PeV}$ are thought to have Galactic origin. A direct way to prove it is to identify the origin (PeVatrons) of these high-energy CRs. The main drawback is that the CRs detected at the Earth, being charged particles, do not point back to the sources, because their trajectories are bent by the Galactic magnetic fields, unless they have extremely high-energies (around $\mathrm{EeV}$ ). However, thanks to the gamma-ray radiation produced in the interactions of the CRs, the detection of PeVatrons with the current gamma-ray observatories is plausible. For instance, the first detection of a PeVatrons, at the center of the Milky Way, was achieved by the High Energy Stereoscopic System (H.E.S.S.) telescopes [1].

The High Altitude Water Cherenkov Gamma-Ray Observatory (HAWC) is a cosmic and gamma-ray detector located in the slope of volcano Sierra Negra, next to Puebla, in central Mexico. HAWC has three main properties that make it a perfect instrument to survey the Galactic gammaray sky searching for PeVatrons: good TeV sensitivity, large field of view (2 sr), and high duty cycle (>90\% uptime).

In this work we present the analysis of the region around 2HWC J1825-134 which has been claimed by HAWC as one of the most energetic sources in the Galaxy [2] and a PeVatron candidate.

\section{HAWC observation of the 2HWC J1825-134 region}

2HWC J1825-134 was identified as one of the brightest sources in the second HAWC catalog released two years ago [3]. Due to the complexity of the region around J1825, detailed analysis was out of the scope of the catalog. The present contribution includes a more exhaustive multi-source analysis of the region, using a dataset which doubles the lifetime of the one used in the second HAWC catalog.

Moreover, the analysis also benefits from the recent developments in the HAWC energy reconstruction. In particular, the energy resolution of the events detected in the observatory have improved thanks to the implementation of two independent energy-estimation methods, which allow the measurement of gamma-ray energies beyond $100 \mathrm{TeV}$ [4]. This new energy estimation methods have been used to create a high-energy catalog of sources emitting above 50 and $100 \mathrm{TeV}$ that will be presented in this conference [2]. Among those sources 2HWC J1825-134 which stands out as the one with the greatest statistical significance.

\subsection{Source modeling}

The modelling and spectral fitting in this work was done using The Multi-Mission Maximum Likelihood framework (3ML) [5]. The model adopted in the analysis takes as a region of interest (ROI) a $2.5^{\circ}$ disk around 2HWC J1825-134, and contains an extended Gaussian-shaped gammaray source at the position of $2 \mathrm{HWC}$ J1825-134, with a power law with an exponential cut-off as the gamma-ray spectrum. The model also includes the known VHE gamma-ray source LS 5039 at $\left[\mathrm{RA}=276.56^{\circ}, \mathrm{Dec}=-14.83^{\circ}\right]$, since it falls within the ROI, as a point-like source following a simple power law. Finally, a Galactic diffuse emission (GDE) component [6] with a Gaussian shape along the Galactic latitude (b) and peaked at $b=0^{\circ}$ was also added to the model. The spectral shape of the GDE was a simple power law. For simplicity, and based on previous HAWC studies, 
the Gaussian width of the GDE component was fixed to $1^{\circ}$ and the spectral index to 2.75 , leaving only the normalization as a free parameter for the global fit. It was tested that the impact on the other sources in the model, after freeing all the GDE parameters, was less than $10 \%$. In any case, the effect was included as a part of the systematic uncertainties. It is important to notice that in the GDE component includes not only the emission from the interactions between CRs and interstellar gas/radiation fields, but also all the emission from sources below the HAWC detection threshold.

Since H.E.S.S. observations of the same region claim two sources $[7,8]$ instead of one, a HAWC model having two sources instead of the original 2HWC J1825-134 was also tested. This possibility turned out to be statistically preferred $(>5 \sigma)$ with respect to the initial hypothesis of only one source. Therefore, for the rest of the analysis two sources with names HAWC J1825137 at $\left[\mathrm{RA}=(276.35 \pm 0.03)^{\circ}, \mathrm{Dec}=(-13.73 \pm 0.05)^{\circ}\right]$ and HAWC J1826-129 at $[\mathrm{RA}=(276.51 \pm$ $\left.0.03)^{\circ}, \operatorname{Dec}=\left(-12.93 \pm 0.05^{\circ}\right)\right]$ are considered. These sources claimed in this work by HAWC have an angular distance with respect to the H.E.S.S. sources of approximately $0.1^{\circ}$ degrees each, which means that they are associated.

The HAWC significance map of the region is shown in Fig. 1 left. Considering all the pixels in the residual significance map ${ }^{1}$ within $2^{\circ}$ from the center of 2 HWC J1825-134, the test statistic (TS) values from the residual map are represented as a histogram in Fig. 1 right. This histogram helps to test the validity of the employed model, since it should follow a Gaussian distribution centered at 0 with a width of 1 , typical of the background fluctuations, when data and model match.
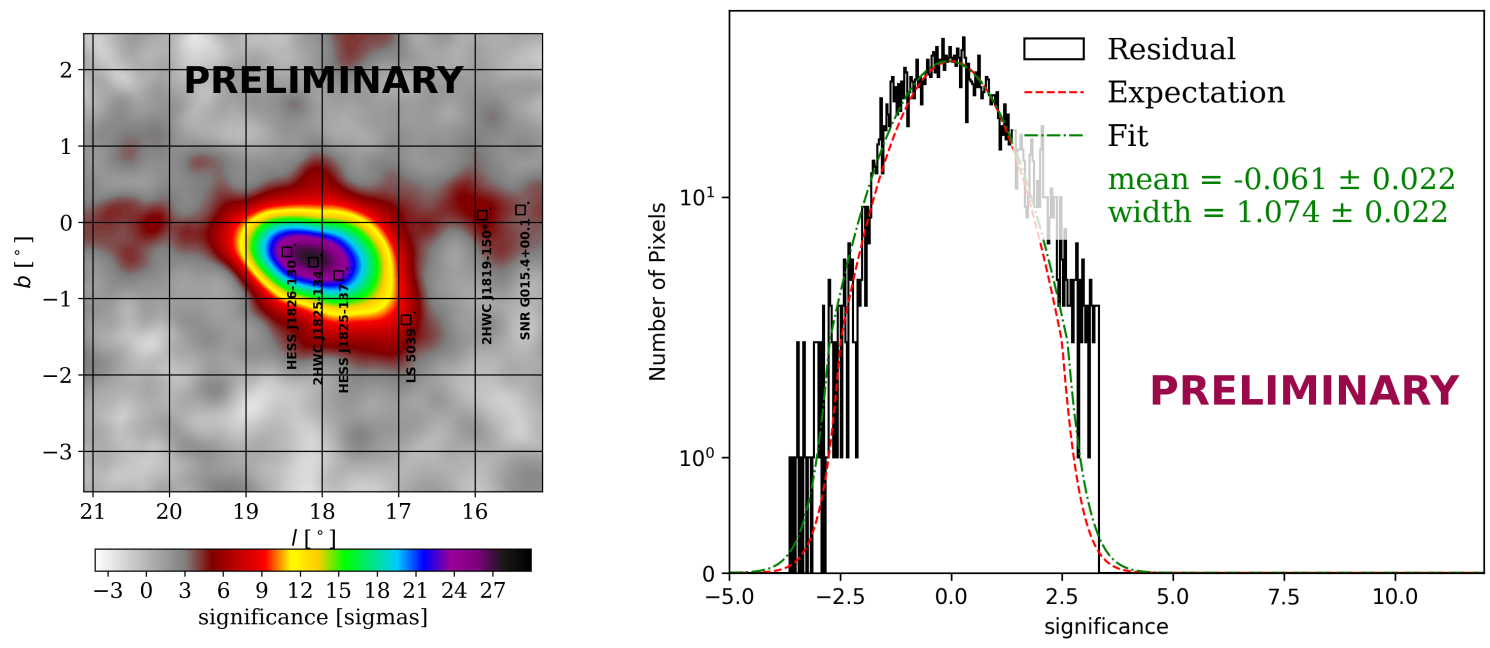

Figure 1: Significance map around the 2HWC J1825-134 region (left). The labels in the map come from the TeVCat database[9]. Histogram of the TS from the residual significance map (right), i.e., when the fitted sources and GDE were added to the background model.

\subsection{Spectral studies}

The gamma-ray spectrum for modeling the sources is a simple power law with and exponential cut-off (see Eq. 2.1).

\footnotetext{
${ }^{1}$ The residual map significance map is made considering that the modeled sources are added to the background model
} 


$$
\phi(E)=\phi_{0}\left(\frac{E}{E_{0}}\right)^{-\alpha} \exp \left(\frac{-E}{E_{\text {cut }}}\right)
$$

where $\mathrm{E}$ is the gamma-ray energy, $\mathrm{E}_{0}$ is the pivot energy which was chosen to be $18 \mathrm{TeV}$ to minimize correlations between the free parameters in the fit, $\alpha$ is the spectral index, $\mathrm{E}_{\text {cut }}$ is the energy cut-off, $\phi(E)$ represents the flux at a given energy, and $\phi_{0}$ is the flux at the pivot energy.

The results of the spectral fit using a minimum likelihood method with 3ML are in Table 1.

\begin{tabular}{|c|c|c|c|c|}
\hline Source Name & Gaussian width $\left[^{\circ}\right]$ & $\phi_{0}\left[\mathrm{~cm}^{-2} \mathrm{TeV}^{-1} \mathrm{~s}^{-1}\right]$ & $\alpha$ & $\mathrm{E}_{\text {cut }}[\mathrm{TeV}]$ \\
\hline HAWC J1825-137 & $0.34 \pm 0.03$ & $2.9_{-0.6}^{+0.7} \times 10^{-14}$ & $2.16 \pm 0.12$ & $60_{-20}^{+30}$ \\
\hline HAWC J1826-129 & $0.23 \pm 0.03$ & $2.6_{-0.8}^{+1.1} \times 10^{-14}$ & $1.5 \pm 0.3$ & $33_{-12}^{+20}$ \\
\hline
\end{tabular}

Table 1: Values of the spectral parameter fitted.

The fit of the GDE component included in the model gives a normalization flux at $10 \mathrm{TeV}$ of $(2.0 \pm 0.2) \times 10^{-14} \mathrm{~cm}^{-2} \mathrm{TeV}^{-1} \mathrm{~s}^{-1} \mathrm{sr}^{-1}$. This flux corresponds to around $15 \%$ of the flux of the sources HAWC J1825-137 and HAWC J1826-129. It is also worth to mention that LS 5039 shows a statistical significance of $3 \sigma$ in the combined fit, so it is not considered a firm detection by HAWC.

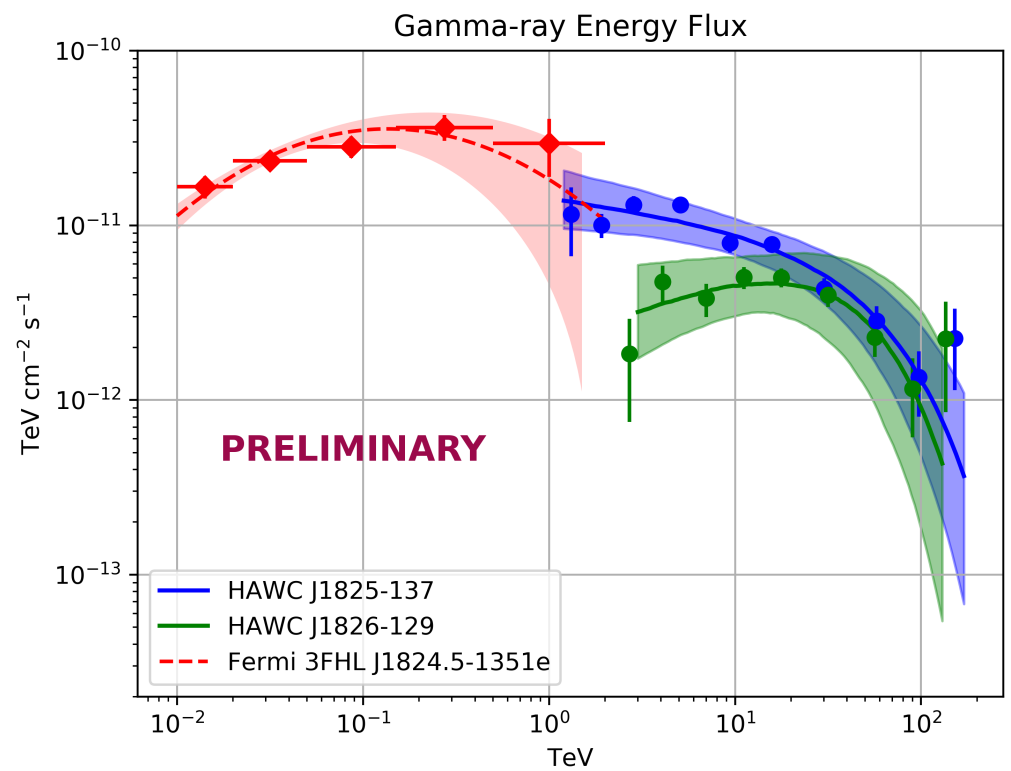

Figure 2: Gamma-ray energy flux of HAWC J1825-137 (blue) and HAWC J1826-129 (green). HAWC flux points were computed as in [4]. Fermi-LAT flux points (red) come from the 3FHL catalog [11].

From the spectral plot in Fig. 2 we can see that HAWC J1825-137 is dominant with respect to HAWC J1826-129 at low energies but at energies above $30 \mathrm{TeV}$ the flux of both sources becomes comparable. The cutoff energies are 60 and $30 \mathrm{TeV}$, and the gamma-ray emission of both sources 
extend beyond $100 \mathrm{TeV}$. This high-energy emission and the physical implications will be covered in the conference by dedicated contributions $[2,10]$. Also noticeable is the hard spectrum measured for HAWC J1826-129, which is compatible with the measurement reported by H.E.S.S. [8].

The results of the fit can be seen graphically in Fig. 2. Fermi-LAT points from the 3FHL catalog [11] for J1824.5-1351e (corresponds to HAWC J1825-137) where also added. From the plot one can see that HAWC observation is compatible with Fermi flux points at the energy where both measurements connect, around 1TeV. In the case of HAWC J1826-129, Fermi-LAT points have large uncertainties so were not included in the plot.

Finally, we studied the systematic effects that could impact the results of the fit. The study was performed in a similar fashion as in [4]. From this study we concluded that the impact on the fit parameters in our analysis are up to $50 \%$ in flux, $15 \%$ in spectral index. For the cutoff energy the uncertainties are quite asymmetric, and the impact can go up to $100 \%$ in the positive interval, and up to $20 \%$ in the negative interval.

\subsection{Morphology studies}

The radial profile of the gamma-ray emission from HAWC J1825-137 and HAWC J1826-129 were computed and compared with the prediction of the Gaussian shaped morphological model assumed. All the sources in the region were masked, with the exception of the one being analyzed. The results, in Fig. 3, shown a good agreement between the data and the model.
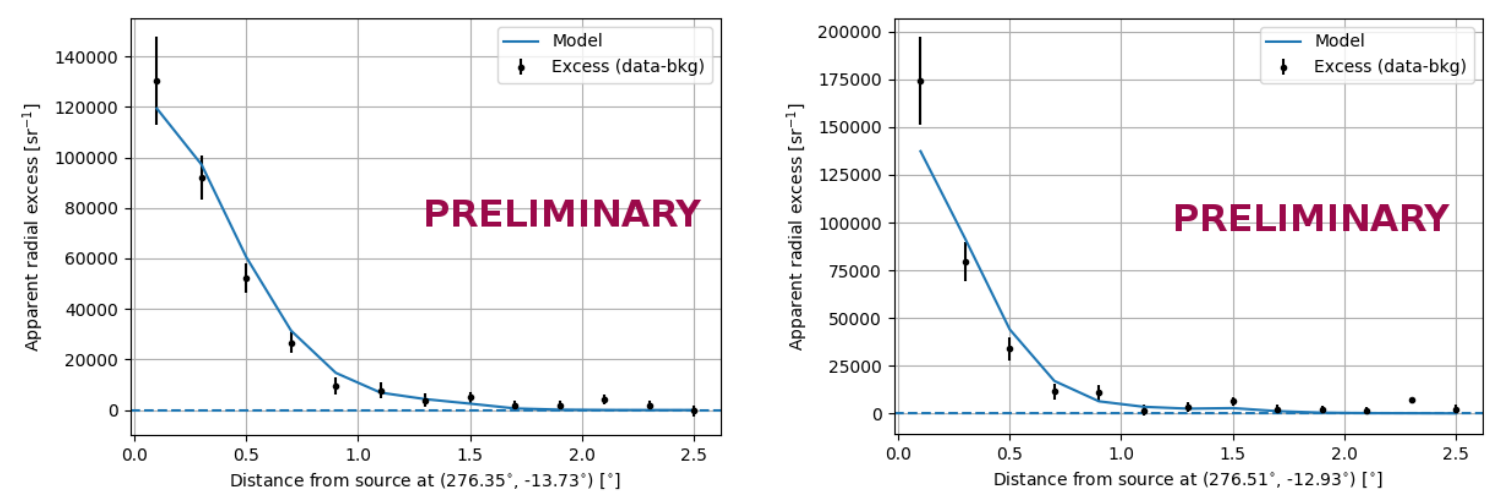

Figure 3: Radial profile of J1825 (left) and J1826 (right). The points are the apparent radial excesses per solid angle. The blue lines represent the expected values for the Gaussian morphological model used in the analysis.

However, motivated by H.E.S.S. observations [7], with a better angular and energy resolution than HAWC, of the largest source in the region, we did some extra checks of HAWC J1825-137 morphology. In particular, we tested morphology based on a disk shaped source. However, the Gaussian shaped morphology was clearly $(>5 \sigma)$ preferred.

Nevertheless, it is worth to mention that even if the Gaussian model used seems to reproduce well the data, looking at the residual map in Fig. 4 left, one can notice a 2 sigma fluctuation close to the center of the region being studied, which may indicate that the model tested is still not elaborated enough to accurately reproduce the emission. 
HAWC J1825-137 was also tested looking for anisotropic emission. In this regard, the excess per steradian in small regions around the center was calculated. Each region was defined by an opening angle of $20^{\circ}$, and covered up to an angular distance of $2^{\circ}$. The $0^{\circ}$ rotation angle direction was defined using the HAWC J1825-137 as center, increasing RA, and equal declination. The rotation steps were done clock-wise. Again, the other sources in the ROI were masked to not bias the results. Fig. 4 shows that, considering the large statistical uncertainties, most of the emission around HAWC J1825-137 is compatible with isotropy, except a region around 270-300 degrees, which corresponds to the high significance blob in the lower left part of the map in Fig. 4 left. Although there is no TeV gamma-ray source known in that region, Fermi-LAT observations point to a possible emission in the vicinity of 1825 [12], which may correlate with this high significance blob observed by HAWC. Current HAWC observations, around $4 \sigma$, are not significant enough to claim the emission, but it will be confirmed or excluded with upcoming data.
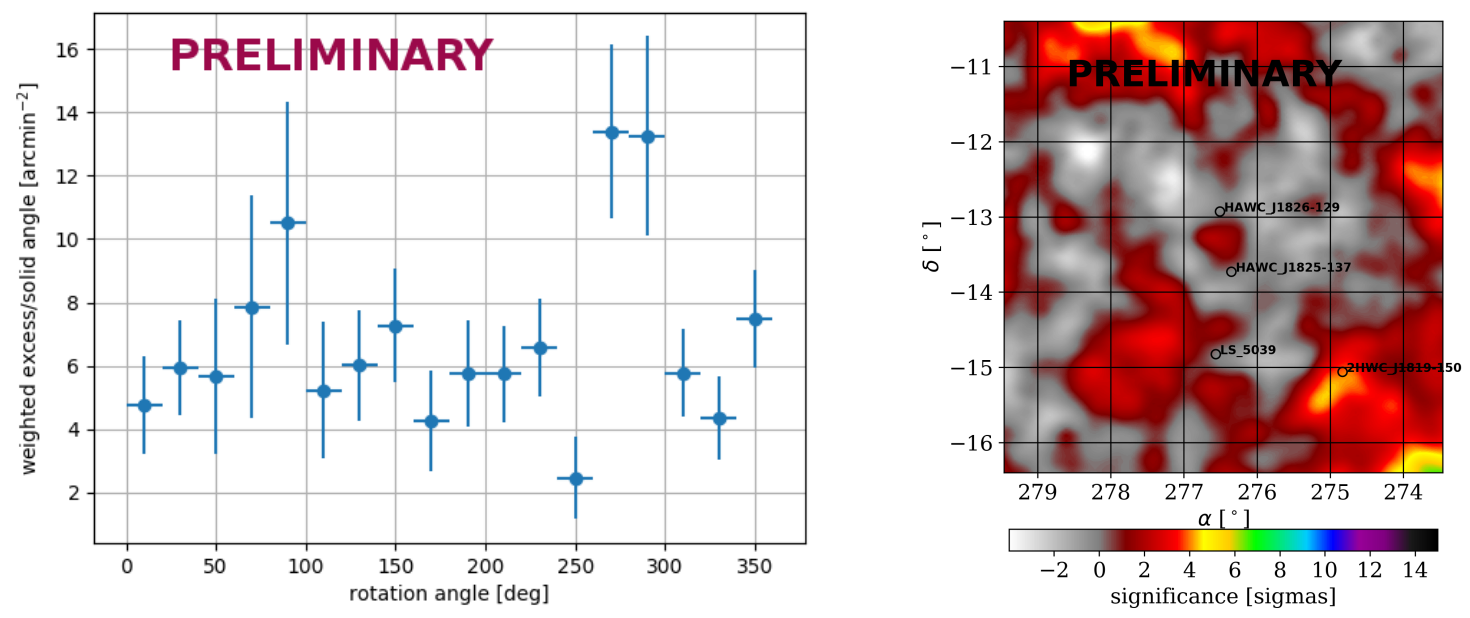

Figure 4: Plot showing the excess gamma-ray emission depending on the orientation around HAWC J1825137 (left). Residual map around the region studied, in equatorial coordinates (right).

\subsection{Energy dependent morphology}

The radial profiles of both HAWC J1825-137 and HAWC J1826-129 were studied for 3 different energy bins of $1-10 \mathrm{TeV}, 10-56 \mathrm{TeV}$ and $56-316 \mathrm{TeV}$. The fit to a Gaussian gives a width of 0.38 $\pm 0.04,0.29 \pm 0.04$, and $0.21 \pm 0.07$ respectively for HAWC J1825-137. Therefore, the observed trend is the higher the energy, the smaller the size. However, due to the large uncertainties, an energy dependent morphology with enough statistical significance cannot be claimed yet.

The same study for HAWC J1826-129, the smallest of the two sources studied, was even more challenging since the size of the source was comparable with the HAWC angular resolution. The relative statistical uncertainties when measuring the Gaussian width were larger, and the test was inconclusive.

\subsection{Particle spectra}

The particle spectrum that is responsible for the gamma-ray emission detected by HAWC is a crucial piece of information to understand the nature of the sources. In this analysis we used 
Naima [13], which is a Python package for computation of non-thermal radiation from relativistic particle populations, to derive that spectrum.

Knowing that HAWC J1825-137 is a PWN associated with pulsar PSR J1826-1334 at 4kpc [14], we assumed that the particle acceleration process was leptonic and used an Inverse Compton (IC) model [15]. The chosen parametrization was [16] as in [7]. When fitting HAWC data with Naima, a particle spectrum following a simple power law with an energy cut-off was preferred over other spectra tested (simple power law and broken power law). However, when adding the Fermi-LAT 3FHL flux points to the fit, then a broken power law was preferred with the spectral parameters: $\alpha_{1}=1.3_{-0.4}^{+0.3}, \alpha_{2}=3.01 \pm 0.04, \mathrm{E}_{\text {break }}=0.75 \pm 0.18 \mathrm{TeV}$, and $\mathrm{W}_{e} \sim 5.0 \times 10^{48}$ erg. Where $\mathrm{W}_{e}$ is the energy in electrons between energies from $1 \mathrm{GeV}$ to $100 \mathrm{PeV}$.

The case of HAWC J1826-129 is more interesting since it is unidentified, and the particle fit can reveal its nature. This source has been associated to the pulsar PSR J1826-1256 at a distance of $1.5 \mathrm{kpc}$ [14], but also to giant molecular clouds [17] at $4 \mathrm{kpc}$. Therefore, either leptonic or hadronic models could be used to explain the emission in gamma-rays. Considering this, two Naima models were fitted to HAWC data. First an IC model based on [15] with a parametrization from [18]. The second model accounts for gamma-rays produced after neutral pion decay [19] with a particle density of $\mathrm{n}_{h}=550 \mathrm{~cm}^{-3}$. In both cases a simple power law with energy cutoff was assumed as the particle spectrum. The results hint, with roughly $2 \sigma$, than the IC model is preferred over the $\pi_{0}$ decay one, with the spectral parameters: $\alpha=1.2 \pm 0.4, \mathrm{E}_{\text {cut }}=51_{-11}^{+14}, \mathrm{~W}_{e} \sim 10^{46} \mathrm{erg}$.

The particle flux spectrum for the best fits (IC for HAWC J1825-137 plus Fermi 3FHL J1824.51351e, and IC for HAWC J1826-129) is shown in Fig. 5.

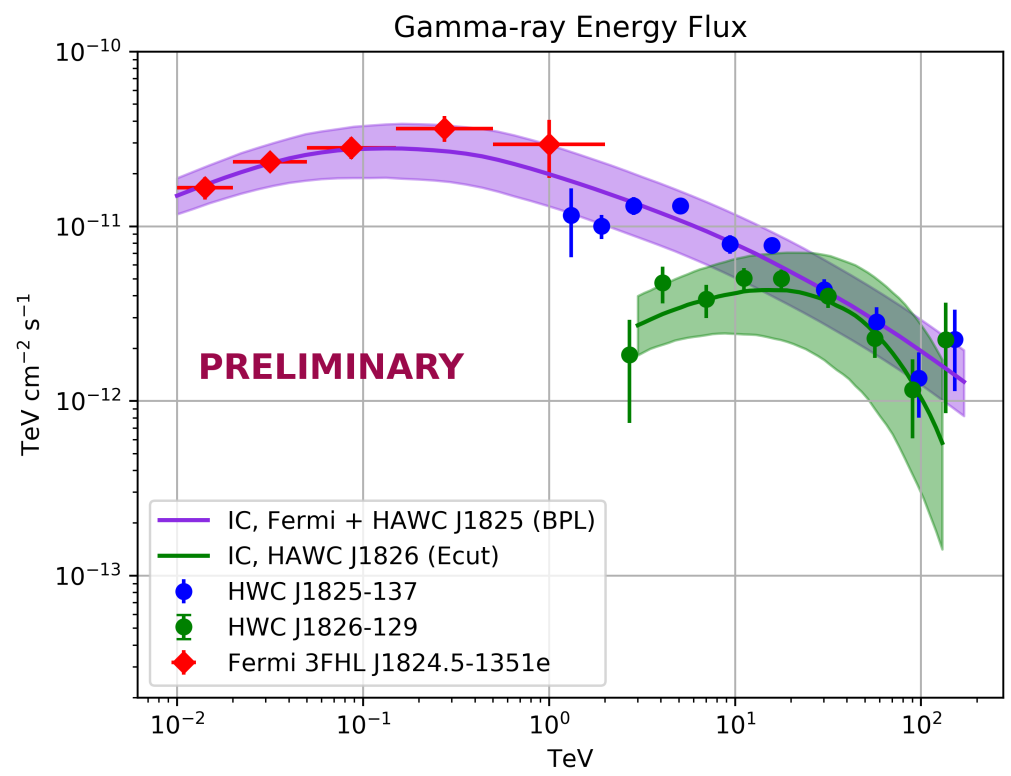

Figure 5: Gamma-ray energy flux spectrum derived from the particle spectral fit with the Naima package. The violet curve represent the fit to HAWC J1825-137 together with Fermi 3FHL J1824.5-1351e. The green curve represents the HAWC J1826-129 fit using only HAWC data. 


\section{Conclusion/Discussion}

In this contribution we have presented a detailed study of the region around 2HWC J1825-134 showing that it contains two sources, HAWC J1825-137 and HAWC J1826-129. Both show a fair agreement when modeled with a Gaussian shaped morphology. In the case of HAWC J1825-137 there is also an indication for an energy dependent morphology, but more data will be needed to get a firm conclusion with enough significance. Moreover, from the study of its morphology, it was observed a nearby emission that may be correlated with an emission observed by Fermi-LAT [12].

We also discussed the particle spectrum producing the gamma-ray emission observed by HAWC. We conclude that an inverse compton emission from an electron population with a broken power-law energy spectrum is able to explain together Fermi-LAT and HAWC emission for the case of HAWC J1825-137. For the case of HAWC J1826-129, which is unidentified, the comparison of a $\pi_{0}$ model with respect to a IC model seems to favors the latter at $2 \sigma$.

It is expected that additional data from the recently completed HAWC outrigger array will be added soon to the analysis. The outrigger data will increase significantly the high-energy statistics and therefore, will be key to improve our understanding of the J1825 complete region.

\section{References}

[1] H.E.S.S. Coll., Nature, 531 (2016) 476,

[2] K. Malone, for the HAWC collaboration, First HAWC Spectra of Galactic Gamma-ray Sources Above $100 \mathrm{TeV}$ and the Implications for Cosmic-ray Acceleration, in Proceedings for the ICRC 2019.

[3] A.U. Abeysekara et al., Astrophys J.843 (2017) 40,

[4] A.U. Abeysekara et al., submitted to Astrophys J., https://arxiv.org/abs/1905.12518

[5] G. Vianello et al., The Multi-Mission Maximum Likelihood framework (3ML) (2015), https://github.com/giacomov/3ML

[6] C. D. Rho et al. for the HAWC Collaboration, PoS (ICRC2 017) 741 (2017).

[7] H.E.S.S. Coll., Astron. Astrophys., 621 (2019) A116,

[8] E. O. Angüner, for the H.E.S.S. Collaboration, PoS ( ICRC2 017) 686 (2017).

[9] TeVCat catalog, http://tevcat.uchicago.edu

[10] J. T. Linnemann, for the HAWC collaboration, Highest Energy Photons Ever Observed and HighEnergy Crab Spectrum, in Proceedings for the ICRC 2019.

[11] Fermi-LAT Coll., Astrophys. J. Suppl. S., 232 (2017) 18,

[12] M. Araya et al, Mon. Not. R. Astron. Soc., 485, May 2019, 1001-1007,

[13] V. Zabalza, PoS ( ICRC2015) 922 (2016).

[14] R. N. Manchester, G. B. Hobbs, A. Teoh and M. Hobbs, Astron. J., 129 (2005) 1993-2006,

[15] D. Khangulyan et al., Astrophys J.783 (2014) 100,

[16] C. C. Popescu et al., Mon. Not. R. Astron. Soc., 470 (2017), 2539

[17] F. Voisin et al., Mon. Not. R. Astron. Soc., 458 (2016), 2813

[18] S. Vernetto and P. Lipari, Phys. Rev. D94 (2016) 063009,

[19] E. Kafexhiu et al., Phys. Rev. D90 (2014) 123014 , 